\title{
Structure of Campylobacter jejuni Lipopolysaccharides Determines Antiganglioside Specificity and Clinical Features of Guillain-Barré and Miller Fisher Patients
}

\author{
C. W. Ang,${ }^{1,2,3 *}$ J. D. Laman, ${ }^{1}$ H. J. Willison, ${ }^{4}$ E. R. Wagner, ${ }^{4}$ H. P. Endtz ${ }^{3}$ M. A. De Klerk, ${ }^{1,2}$ \\ A. P. Tio-Gillen, ${ }^{1,2}$ N. Van den Braak, ${ }^{3}$ B. C. Jacobs,,${ }^{1,2}$ and P. A. Van Doorn ${ }^{1}$ \\ Departments of Neurology, ${ }^{1}$ Immunology, ${ }^{2}$ and Microbiology and Infectious Diseases, ${ }^{3}$ Erasmus University/Academic Hospital \\ Dijkzigt Rotterdam, Rotterdam, The Netherlands, and Department of Neurology, Southern General Hospital, \\ Glasgow G51 4TF, Scotland ${ }^{4}$
}

Received 22 August 2001/Returned for modification 5 November 2001/Accepted 21 November 2001

\begin{abstract}
Ganglioside mimicry in the lipopolysaccharide (LPS) fraction of Campylobacter jejuni isolated from GuillainBarré syndrome (GBS) and Miller Fisher syndrome (MFS) patients was compared with isolates from patients with an uncomplicated enteritis. The antibody response to $C$. jejuni LPS and gangliosides in neuropathy patients and controls was compared as well. LPS from GBS and MFS-associated isolates more frequently contained ganglioside-like epitopes compared to control isolates. Almost all neuropathy patients showed a strong antibody response against LPS and multiple gangliosides in contrast to enteritis patients. Isolates from GBS patients more frequently had a GM1-like epitope than isolates from MFS patients. GQ1b-like epitopes were present in all MFS-associated isolates and was associated with anti-GQ1b antibody reactivity and the presence of oculomotor symptoms. These results demonstrate that the expression of ganglioside mimics is a risk factor for the development of post-Campylobacter neuropathy. This study provides additional evidence for the hypothesis that the LPS fraction determines the antiganglioside specificity and clinical features in postCampylobacter neuropathy patients.
\end{abstract}

Campylobacter jejuni infection is the most frequent triggering event in postinfectious neuropathies such as the GuillainBarré syndrome (GBS) and Miller Fisher syndrome (MFS) $(13,23,29)$. The exact pathogenesis of post-Campylobacter neuropathy is unknown, but molecular mimicry between bacterial glycoconjugates and peripheral nerve gangliosides has been implicated (32). Cross-reactive antibodies between $C$. jejuni lipopolysaccharides (LPS) and gangliosides have been identified in GBS and MFS patients $(12,20)$. The specificity of this cross-reactive antiganglioside-LPS response is associated with different disease profiles. Antibody reactivity against GM1, GM1b, and GalNAc-GD1a is associated with pure motor GBS $(2,33)$, and anti-GQ1b antibody reactivity has a strong association with oculomotor symptoms and ataxia (15).

Ganglioside mimics have been identified in C. jejuni LPS by biochemical and serological methods (17) (Fig. 1). Mass spectometry revealed the presence of GM1- and GD1a-like structures in LPS from GBS patients $(22,36)$ and GD3-like structures in MFS-associated LPS (25). Serological studies with ganglioside-binding toxins and monoclonal antiganglioside antibodies confirmed these findings $(26,37)$. Most $C$. jejuni isolates from GBS and MFS patients that were investigated by biochemical and/or serological methods exhibit ganglioside mimicry, but this may have been caused by selection bias. Little information is available concerning the expression of ganglioside mimics in isolates from uncomplicated $C$. jejuni enteritis

\footnotetext{
* Corresponding author. Mailing address: Department of Medical Microbiology and Infectious Diseases, Erasmus University Medical Centre Rotterdam, Dr Molewaterplein 40, 3015 GD Rotterdam, The Netherlands. Phone: 31-10-463-3867. Fax: 31-10-463-3857. E-mail: Ang@bacl.azr.nl.
}

patients compared to neuropathy-associated isolates (26). Furthermore, detailed studies on antibody responses to glycolipid antigens such as LPS and gangliosides in $C$. jejuni enteritis patients have been limited.

The aim of the present study was to investigate whether there is a difference in the expression of ganglioside mimics in unrelated $C$. jejuni isolates from GBS and MFS patients compared to $C$. jejuni isolates from uncomplicated enteritis patients. In addition, we studied the antibody response to LPS and gangliosides in culture-proven Campylobacter-associated neuropathy patients and control enteritis patients to investigate host-related differences in the immune response to glycolipid antigens.

\section{MATERIALS AND METHODS}

Patients and isolates. We collected 18 GBS- or MFS-related C. jejuni isolates from patients in The Netherlands and Belgium between 1991 and 1998 (7). Two isolates were cultured from the diarrheal stools of two family members of a GBS patient. The GBS patient remained culture negative but showed a serological response highly suggestive of a recent $C$. jejuni infection (1). Only one of these strains was included in the analysis, leaving the total number of neuropathy associated strains at 17. Acute-phase serum samples obtained within one week of the onset of symptoms, clinical information on oculomotor symptoms, and ataxia were available for 16 patients. All GBS patients fulfilled the diagnostic criteria (4), and all MFS patients had oculomotor symptoms and ataxia in the absence of limb weakness. Fourteen of the GBS and MFS patients had suffered a period of diarrhea during the weeks before the development of neurological symptoms; four patients did not report a gastrointestinal illness. Two GBS patients had oculomotor symptoms in addition to their limb weakness. All but two neuropathy patients had immunoglobulin A ( $\operatorname{IgA})$ and/or $\operatorname{IgM}$ serum antibodies against a protein extract of $C$. jejuni, indicative of a recent infection (9). One of the remaining two patients had high-titer $\operatorname{IgG}$ anti-C. jejuni protein antibodies and a history of diarrhea. Twenty-three control $C$. jejuni isolates were obtained from patients with an uncomplicated $C$. jejuni enteritis without neurological symptoms. Of 15 of these control patients, serum samples taken in the first week of the 


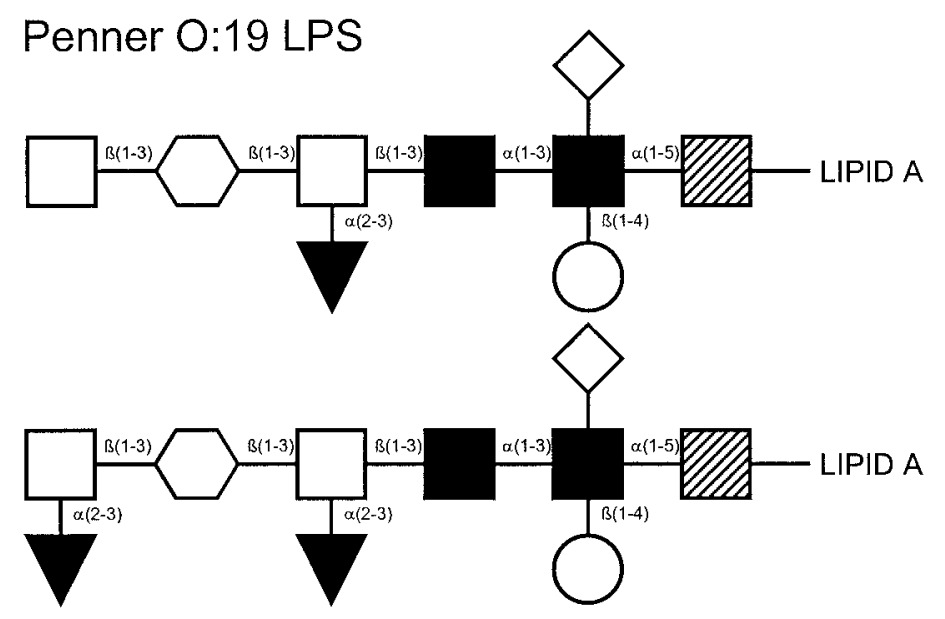

GM1

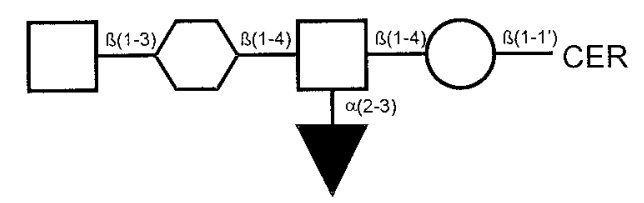

GD1a
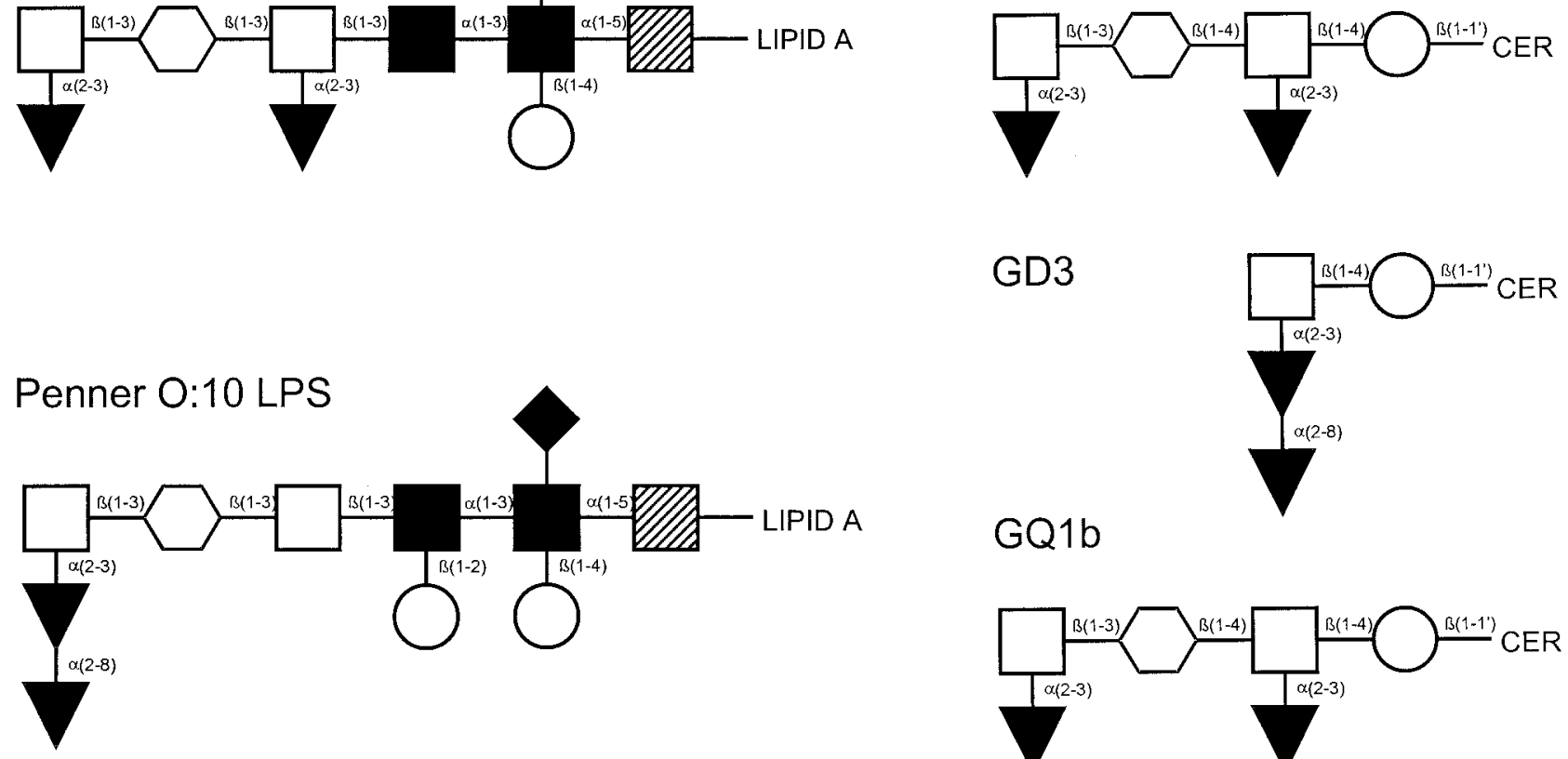

GQ1b

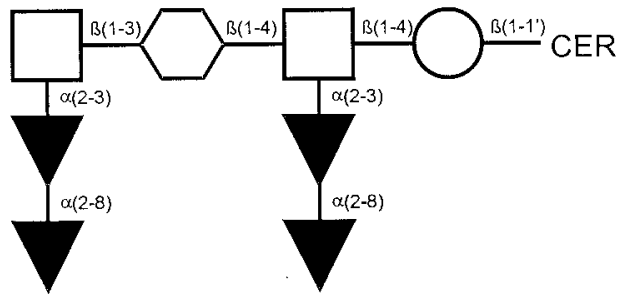

FIG. 1. Structure of $C$. jejuni LPS types and the corresponding ganglioside mimics. Symbols: $\square, N$-acetylgalactosamine; $\square$, galactose; $\bigcirc$, glucose; $\boldsymbol{\nabla}$, sialic acid; $\diamond$, (phosphoryl)ethanolamine; $\bullet$-acetylglucosamine; $\mathbf{\square}$, heptose; $\mathbb{2}$, 3-deoxy-D-manno-octulosonic acid; $\boldsymbol{\bullet}$, dideoxyglucose; $\bullet$, 2-aminoethyl phosphate.

disease were available. All control isolates were collected between 1990 and 1999 from sporadic enteritis patients. All control enteritis patients had suffered from diarrhea. Based on a limited number of patients, there were no differences between the neuropathy and the control enteritis patients with respect to the duration of diarrhea. The seasonal distribution of neuropathy associated and control isolates was the same with a broad summer peak. As additional controls for serotyping of $C$. jejuni isolates, we used reference strains from which the core oligosaccharides have been shown to contain a GM1 mimic (Penner O:19, CCUG 10950) (5), a GD3 mimic (Penner O:10, CCUG 10943) (19), or no ganglioside mimic (Penner O:3, CCUG 10937) (6) (Fig. 1).

Serology. Acute-phase serum samples were tested for $\operatorname{IgM}$ and $\mathrm{IgG}$ antibody reactivity against asialo-GM1 (GA1), GM1, GM2, GD1a, GD1b, GD3, and GQ1b by enzyme-linked immunosorbent assay (ELISA) and confirmed by thinlayer chromatography as described previously (10). All glycolipids were obtained from Sigma (St. Louis, Mo.), except GQ1b (Biocarb, Lund, Sweden). LPS fractions from all isolates were isolated with the hot-phenol-water method and processed as described previously (12). All LPS fractions showed a dense band migrating at 8 to $15 \mathrm{kDa}$ after electrophoresis on a polyacrylamide gel and silver staining (Novex, San Diego, Calif.), indicating the presence of LPS (5). For the detection of anti-LPS reactivity, serum samples were tested by ELISA at dilutions of 1:100 and 1:1,000 as described previously (11). Samples were considered positive when the corrected optical density value was $>3$ standard deviations of the mean optical density for a group of 12 healthy controls without serological evidence of a recent $C$. jejuni infection.

To assess serum samples for cross-reactive antibodies between LPS and gangliosides, serum was incubated with $C$. jejuni LPS conjugated to Octyl-Sepharose CL4B beads. Then, $250 \mu \mathrm{g}$ of LPS was added to $1 \mathrm{ml}$ of Octyl-Sepharose CL4B in methanol-water $(1: 1[\mathrm{vol} / \mathrm{vol}])$ containing $0.1 \mathrm{M} \mathrm{KCl}$ and mixed for $1.5 \mathrm{~h}$. After several washes with phosphate-buffered saline ( $\mathrm{pH} 7.8$; PBS), serum samples were incubated with LPS-Sepharose conjugates for $5 \mathrm{~h}$ at $4^{\circ} \mathrm{C}$. After centrifugation, absorbed serum samples were tested for anti-LPS and antiglycolipid reactivity as described above. Serum samples that were incubated with beads coupled to LPS from the Penner O:3 serostrain that does not contain a ganglioside mimic (6) or uncoupled beads served as controls.

Serological typing of $\boldsymbol{C}$. jejuni LPS. (i) Polyclonal serum panel. Purified LPS was tested in an ELISA-based system for the presence of ganglioside-like epitopes with a panel of ganglioside-reactive sera divided into four groups. Some, but not all, of the samples were from culture-positive GBS or MFS patients. The first group (GM1) consisted of 10 serum samples with IgG anti-GM1 reactivity. The second group (GQ1b) consisted of 10 serum samples with IgG anti-GQ1b reactivity. The third group (Other) consisted of four serum samples with antiganglioside reactivity against GM2 and GD1a. This group was included to detect ganglioside mimics that could not be defined with sera from the GM1 and GQ1b group. The fourth group (HC) consisted of 12 healthy controls without serolog- 
TABLE 1. Antiglycolipid titers and anti-LPS reactivity in GBS and MFS patients

\begin{tabular}{|c|c|c|c|c|c|c|c|c|c|c|c|c|c|c|c|c|}
\hline \multirow{2}{*}{ Condition and patient } & \multicolumn{8}{|c|}{ IgG titer $^{a}$} & \multicolumn{8}{|c|}{$\operatorname{IgM}_{\text {titer }}{ }^{a}$} \\
\hline & GA1 & GM1 & GM2 & GD1a & GD1b & GD3 & GQ1b & LPS & GA1 & GM1 & GM2 & GD1a & GD1b & GD3 & GQ1b & LPS \\
\hline \multicolumn{17}{|l|}{ GBS } \\
\hline GB1 & 1,600 & 800 & - & - & - & - & - & + & 1,600 & 400 & - & - & 100 & - & - & + \\
\hline GB2 & NT & NT & NT & NT & NT & NT & NT & NT & NT & NT & NT & NT & NT & NT & NT & NT \\
\hline GB3 & - & - & - & - & - & - & - & + & - & - & - & - & - & - & - & - \\
\hline GB4 & - & - & - & - & - & - & - & + & - & - & - & - & - & - & - & - \\
\hline GB5 & 400 & - & 100 & - & - & - & - & + & 400 & - & - & - & - & - & - & + \\
\hline GB11 & 200 & 800 & - & - & 3,200 & - & - & + & - & - & - & - & - & - & - & + \\
\hline GB13/14 & 1,600 & 400 & - & - & - & - & - & + & 800 & 200 & - & - & 200 & - & - & + \\
\hline GB15 & - & - & - & - & - & - & - & - & - & - & - & - & - & - & - & - \\
\hline GB17 & 25,600 & 100 & - & - & - & 100 & - & + & 200 & - & - & - & - & - & - & + \\
\hline GB18 & 3,200 & 2,800 & - & - & 1,600 & - & - & + & - & 200 & - & - & - & - & - & + \\
\hline GB21 & 800 & 100 & - & 6,400 & - & - & - & + & - & - & - & - & - & - & - & + \\
\hline \multicolumn{17}{|c|}{ GBS oculomotor symptoms } \\
\hline GB16 & 200 & - & - & - & - & 3,200 & $>102,400$ & + & 100 & - & - & - & - & - & - & + \\
\hline GB19 & - & - & - & - & - & 100 & $>12,800$ & + & 100 & - & - & - & - & - & - & + \\
\hline \multicolumn{17}{|l|}{ MFS } \\
\hline MF6 & - & - & - & - & 100 & 200 & 1,600 & + & - & - & - & - & - & - & - & - \\
\hline MF7 & - & - & - & - & - & - & 400 & + & - & - & - & - & - & - & 200 & + \\
\hline MF8 & - & - & - & - & 200 & 200 & 800 & + & - & - & - & - & 200 & - & - & + \\
\hline MF20 & 1,600 & - & - & - & - & - & 100 & - & 400 & - & - & - & - & - & - & + \\
\hline
\end{tabular}

${ }^{a}$ NT, not tested; +, titer $>100 ;-$, titer $<100$.

ical evidence for a recent $C$. jejuni infection. An LPS was considered to have a GM1-like epitope when at least 3 of 10 serum samples from the GM1 group had an optical density of $>3$ standard deviations above the mean of the healthy controls, with a minimum optical density of 0.300 (to avoid false-positive samples due to the low levels of anti-LPS reactivity seen in healthy control samples). The criteria for LPS bearing a GQ1b-like epitope were similar with the GQ1b panel of sera. An LPS was considered to have an undefined ganglioside-like epitope, which could not be designated GM1-like or GQ1b-like, when the LPS showed reactivity with only one or two serum samples in either the GM1 or the GQ1b group or reactivity with one or more serum samples from the "other" group.

(ii) Toxins, lectin, and MAbs. The specificity of the binding of cholera toxin B subunit (CT), tetanus toxin fragment C (TT), peanut agglutinin (PNA), and the monoclonal antiganglioside antibodies (MAbs) Sm1 (31), Wo1 (21), Ha1rbc (30), CGM3 (8), and EG1 (8) was validated by binding these ligands to GA1, GM1, GM2, GM3, GD1a, GD1b, GD3, GT1b, or GQ1b or to bacterial LPS. Plates (96 well; Nunc Immunosorb, Roskilde, Denmark) were coated with glycolipids as described previously (11). Wells without glycolipids served as controls. Peroxidase-conjugated CT (List Biologicals, Campbell, Calif.; Sigma), biotinconjugated CT (List Biologicals), peroxidase-conjugated TT (List Biologicals), peroxidase-conjugated PNA (Sigma), or the MAbs diluted in PBS-bovine serum albumin were added for $1 \mathrm{~h}$ at room temperature (toxins) or overnight at $4^{\circ} \mathrm{C}$ (MAbs). After being washed with PBS, plates containing peroxidase-conjugated ligands were developed with $o$-phenyldiamine. Biotin-conjugated CT was incubated with peroxidase-conjugated avidin (ABC Kit; Vector, Burlingame, Calif.) for $1 \mathrm{~h}$ at room temperature, followed by washing and development. Plates containing MAbs were incubated with peroxidase-conjugated anti-human or anti-mouse antibodies and washed with PBS before development. Specificity of binding to purified glycolipids was confirmed with a thin-layer chromatography overlay (14).
Statistical analysis. Statistical analysis was performed with Stata verson 6.0 (Stata, College Station, Tex.). Differences in proportions were tested with the Fisher exact test. Binding of toxins, lectin, and MAbs was evaluated with the Mann-Whitney $U$ test. All tests were two-sided. A $P$ value of $<0.05$ was considered significant.

\section{RESULTS}

Patient serology. All but one of the GBS and all MFS patients had IgM and/or IgG antibody reactivity against purified C. jejuni LPS (Table 1). Most serum samples contained reactivity not only against LPS from their autologous isolate but also from other $C$. jejuni isolates. Of 16 neuropathy patients, 13 showed antibody reactivity to a variety of peripheral nerve glycolipids (Table 2). All MFS patients had anti-GQ1b reactivity. Two GBS patients displayed no reactivity against the purified glycolipids, although they both had IgG anti-LPS reactivity. Depletion experiments with LPS conjugated to Sepharose beads demonstrated that the antiglycolipid and anti-LPS antibodies were cross-reactive, not only in patients with antiGM1 and anti-GQ1b antibodies but also in patients with antiGD1a and high-titer anti-GA1 antibodies (data not shown).

Serum from neuropathy patients more frequently contained antibody reactivity against any of the panel of purified neural

TABLE 2. LPS structure and diagnosis of patients with $C$. jejuni enteritis

\begin{tabular}{lcccc}
\hline \multirow{2}{*}{ LPS structure } & \multicolumn{3}{c}{ No. of patients $(\%)$ with diagnosis of: } & \multicolumn{1}{c}{$P$} \\
\cline { 2 - 4 } & GBS $(n=13)$ & MFS $(n=4)$ & Enteritis $(n=23)$ & GBS or MFS vs enteritis \\
\hline GM1-like & $11(85)$ & $2(50)$ & $13(57)$ & 0.32 \\
GQ1b-like & $2(15)$ & $4(100)$ & $2(9)$ & 0.053 \\
Other ganglioside & $8(62)$ & $4(100)$ & $12(52)$ & 0.33 \\
No ganglioside & $1(8)$ & 0 & $8(35)$ & $0.006^{a}$ \\
\end{tabular}

\footnotetext{
${ }^{a}$ Statistically significant.
} 
A. GB11

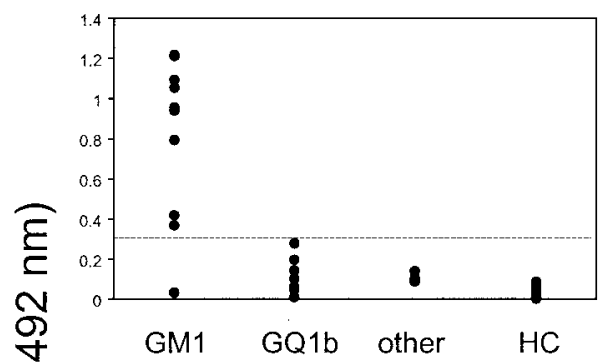

D. Penner O:19

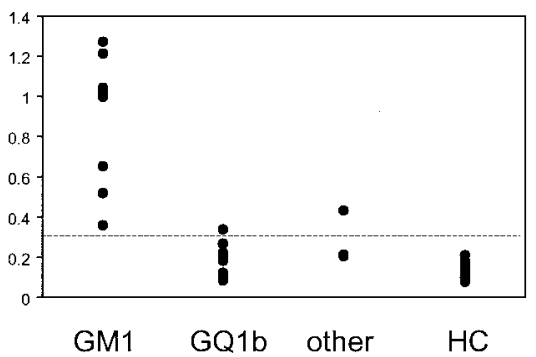

B. GB16

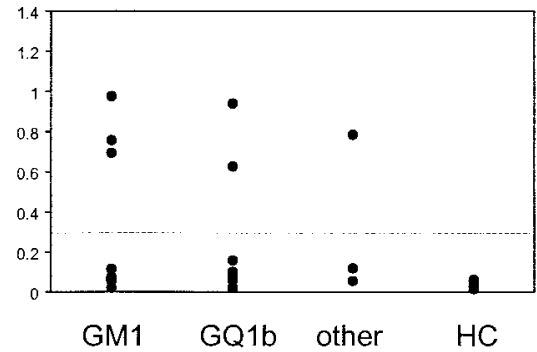

E. Penner O:10

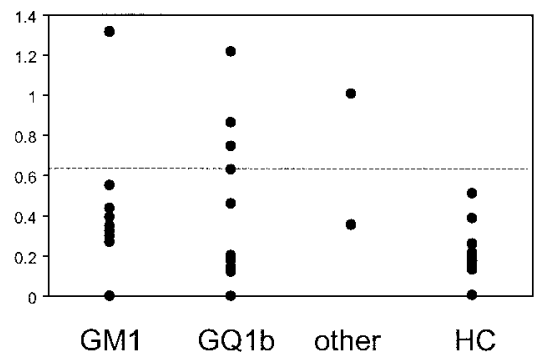

C. MF8

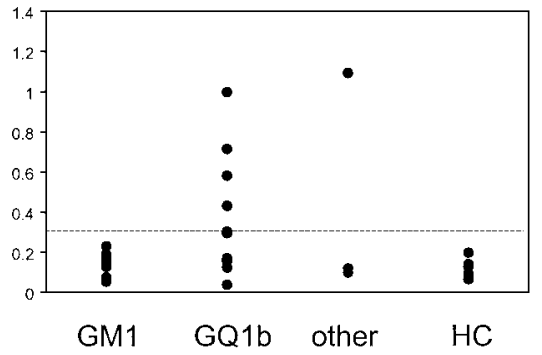

FIG. 2. C. jejuni LPS reacts differentially with antiganglioside antisera. Purified LPSs were tested for reactivity against a panel of serum samples containing antiganglioside reactivity against GM1 and GA1 (GM1), GQ1b and GD3 (GQ1b), or other gangliosides and/or a combination of GA1 and GQ1b ("other"). (A and B) LPS from GBS patients GB11 and GB16 reacted with sera of the GM1 group. In panel B, LPS from GBS patient GB16, who has an overlap of GBS and MFS, reacted with the sera of the GM1, GQ1b, and "other" groups. (C) LPS from MFS patient MF8 reacted predominantly with GQ1b sera. (D) LPS from the O:19 serostrain reacted with sera of the GM1 group but not with those of the GQ1b group, whereas LPS from the O:10 serostrain reacted with the sera from the GQ1b group. (F) LPS from the O:3 serostrain, which does not contain a GM1or GQ1b-like structure, did not react with any of the sera. None of the LPS fractions reacted with sera from the healthy controls (HC). Dashed lines indicate the cutoff values.

glycolipids or $C$. jejuni LPS (15 of 16) than control enteritis patients $(6$ of 15$)(P=0.001)$. In the enteritis group, only one patient had low-titer IgG anti-GM1. Two other patients had anti-GA1 IgG antibodies. Antibody reactivity to gangliosides with two or more sialic acids could not be detected in any of the enteritis patients. Anti-LPS antibodies occurred in three enteritis patients, and one of them also had antibody reactivity against GA1.

Serological typing of $C$. jejuni isolates with GBS or MFS patient sera. Examples of LPS reactivity with antiganglioside antibody containing serum samples and validation of the serum panel with LPS with a known biochemical structure are shown in Fig. 2. The antiganglioside serum panel reacted with all but one LPS from the GBS and MFS isolates, indicating the presence of ganglioside mimics in almost all neuropathy-associated isolates. LPS from most isolates reacted with serum samples from more than one group, suggesting the presence of multiple ganglioside mimics. The Penner serotype was not related to the expression of ganglioside-like epitopes on the LPS. The frequency of GM1-like epitopes was higher in GBS-associated isolates than in MFS or control isolates (Table 2). GQ1b-like epitopes occurred more frequently in MFS-associated isolates than in GBS or control isolates $(P=0.006$, Table 2$)$. Of the control enteritis isolates, $35 \%$ did not react with any of the antiganglioside sera, indicating the absence of ganglioside mimics in these isolates.
To investigate whether the LPS structure predicts the antiganglioside specificity and clinical features in the neuropathy patients, we correlated the results of the LPS typing with these features. In general, the type of ganglioside mimicry in C. jejuni LPS concurred with the antiganglioside specificity and clinical features in the GBS and MFS patients (Table 3).

Serological typing of $C$. jejuni isolates with toxins, lectin, and MAbs. All ligands reacted with purified LPS fractions. None of the toxins, lectin, or MAbs reacted with LPS from the Penner O:3 strain (data not shown). Validation of CT binding, which in its unconjugated form binds highly selectively to GM1 (3), showed differential binding specificity of CT, depending on the conjugated molecule and independent from the concentration of glycolipids (Table 4). Binding of CT and the two antiGA1 and anti-GM1 MAbs correlated well with the serum panel typing system for GM1-like epitopes. CT binding showed a clear difference between GBS- and MFS-related isolates $(P$ $=0.03$, Fig. $3 \mathrm{~A}$ ), although not all GBS-related isolates bound $\mathrm{CT}$ and CT binding was not restricted to GBS patients with anti-GM1 antibodies. In addition, the level of CT binding was higher in neuropathy-associated isolates than in control isolates $(P=0.02)$. The antidisialosyl MAbs clearly distinguished MFS isolates from GBS isolates (Fig. 3B). PNA and TT binding did not have additional value in distinguishing GBS from MFS strains (data not shown). 
TABLE 3. LPS structure, antiganglioside reactivity, and clinical features of GBS and MFS patients

\begin{tabular}{|c|c|c|c|c|c|c|}
\hline \multirow{2}{*}{ Parameter } & \multicolumn{2}{|c|}{$\begin{array}{l}\text { No. of patients (\%) positive } \\
\text { for GM1-like LPS }\end{array}$} & \multirow{2}{*}{$P^{a}$} & \multicolumn{2}{|c|}{$\begin{array}{l}\text { No. of patients }(\%) \text { positive } \\
\text { for GQ1b-like LPS }\end{array}$} & \multirow{2}{*}{$P^{a}$} \\
\hline & Yes $(n=12)$ & No $(n=4)$ & & Yes $(n=6)$ & No $(n=10)$ & \\
\hline \multicolumn{7}{|l|}{ Antiganglioside reactivity } \\
\hline Anti-GA1 & $9(75)$ & $1(25)$ & NS* & $3(50)$ & $7(70)$ & NS* \\
\hline Anti-GM1 & $6(50)$ & 0 & NS* & $1(17)$ & $5(50)$ & NS* \\
\hline Anti-GD3 & $4(33)$ & $1(25)$ & NS* & $4(67)$ & $1(10)$ & $0.04^{*}$ \\
\hline Anti-GQ1b & $4(33)$ & $2(50)$ & NS* & $5(83)$ & $1(10)$ & $0.008^{*}$ \\
\hline \multicolumn{7}{|l|}{ Clinical features } \\
\hline Oculomotor symptoms & $4(33)$ & $2(50)$ & $\mathrm{NS} \dagger$ & $5(83)$ & $1(10)$ & $0.008^{*}$ \\
\hline Ataxia & $3(25)$ & $2(50)$ & $\mathrm{NS} \dagger$ & $4(67)$ & $1(10)$ & $0.04^{*}$ \\
\hline
\end{tabular}

${ }^{a}$ NS, not significant. *, Compared to patients without these antibodies; $\dagger$, compared to patients without these symptoms.

\section{DISCUSSION}

The present study demonstrates that $C$. jejuni isolates from GBS and MFS patients more frequently express ganglioside mimics in their LPS than isolates from uncomplicated enteritis patients. Cross-reactive anti-LPS-antiglycolipid responses can be detected in almost all culture proven cases of $C$. jejunirelated GBS and MFS but not in serum from patients with an uncomplicated enteritis. Differences in LPS structure determine the specificity of the antibody response and thereby the clinical features of the patients.

The GBS patients were highly diverse in their antiganglioside specificity, but almost all had antibody reactivity against the LPS from the isolate with which they had been infected. This serves as further evidence that the antiganglioside antibodies in GBS and MFS patients result from the previous infection with $C$. jejuni. The presence of strong anti-LPS antibody reactivity in patients without reactivity against a panel of gangliosides indicates the presence of antibody reactivity against unknown gangliosides. In contrast to the findings in neuropathy patients, the response to bacterial and neural glycolipids was significantly lower in patients with an uncomplicated $C$. jejuni enteritis, despite the presence of ganglioside mimics in the LPS of some enteritis isolates. This finding underscores the influence of host-related factors such as polymorphisms in immune-response genes, in addition to bacterium-related factors, in the development of neurological symptoms after an infection with $C$. jejuni $(18,26)$. This may either depend on differential expression of ganglioside mimics in $C$. jejuni LPS, due to phase variation in genes encoding glycosyltransferases (16), or to the influence of other immunomodulating factors (28).

For the present study we developed a method with serum samples containing antiganglioside antibody reactivity to detect and define ganglioside-like epitopes on C. jejuni LPS. Serum samples containing antibody reactivity to gangliosides other than GM1 or GQ1b reacted strongly with LPS from several isolates, which indicates the presence of other, as-yetundefined ganglioside mimics. In addition, most LPS fractions reacted with serum samples from more than one serum group, suggesting the presence of multiple ganglioside epitopes on one isolate (34). The data obtained by the serum panel method were confirmed with MAbs and toxins, thus demonstrating the validity of the method.

The lack of CT binding of all MFS-related isolates and some GBS-related isolates is important since CT-binding assays are currently being used to identify isolates that have a higher potential to induce neurological symptoms $(18,24)$. The antiGA1 and anti-GM1 MAbs reacted selectively with a limited number of GBS-related isolates. These results indicate that the use of very specific ligands may not be sufficient to detect the wide variety of ganglioside mimics in C. jejuni LPS. Consequently, GBS patients may have antiganglioside antibodies that are directed against GM1-like gangliosides (e.g., GM1b or GalNAc-GD1a) $(2,35)$. Together, these results indicate that

TABLE 4. Reactivity of toxins, lectin, and MAbs to purified gangliosides and LPSs from C. jejuni serostrains

\begin{tabular}{|c|c|c|c|c|c|c|c|c|c|c|c|c|}
\hline \multirow{3}{*}{$\begin{array}{l}\text { Toxin, lectin, or } \\
\text { MAb }\end{array}$} & \multicolumn{12}{|c|}{ Reactivity $^{a}$ of: } \\
\hline & \multicolumn{9}{|c|}{ Ganglioside } & \multicolumn{3}{|c|}{ LPS } \\
\hline & GA1 & GM1 & GM2 & GM3 & GD1a & GD1b & GT1b & GD3 & GQ1b & Pen O:3 & Pen O:10 & Pen O:19 \\
\hline CT biotin & - & +++ & - & - & - & - & - & - & - & - & - & + \\
\hline CT peroxidase & ++ & +++ & ++ & + & ++ & +++ & - & - & - & - & - & +++ \\
\hline PNA-peroxidase & +++ & - & - & - & - & - & - & - & - & - & - & - \\
\hline TT-peroxidase & - & + & - & - & + & +++ & +++ & - & ++ & - & + & - \\
\hline MAb Sm1 & - & +++ & - & - & - & - & - & - & - & - & - & +++ \\
\hline MAb Wo1 & +++ & +++ & - & - & - & ++ & - & - & - & - & - & +++ \\
\hline MAb Ha1rbc & - & - & - & - & ++ & +++ & - & +++ & +++ & - & + & + \\
\hline MAb EG1 & - & - & - & - & - & - & - & + & ++ & - & + & - \\
\hline MAb CGM3 & - & - & - & - & - & - & - & ++ & ++ & - & ++ & - \\
\hline
\end{tabular}

$a_{-}$, No binding; + , weak binding;,++ moderate binding;,+++ strong binding. 

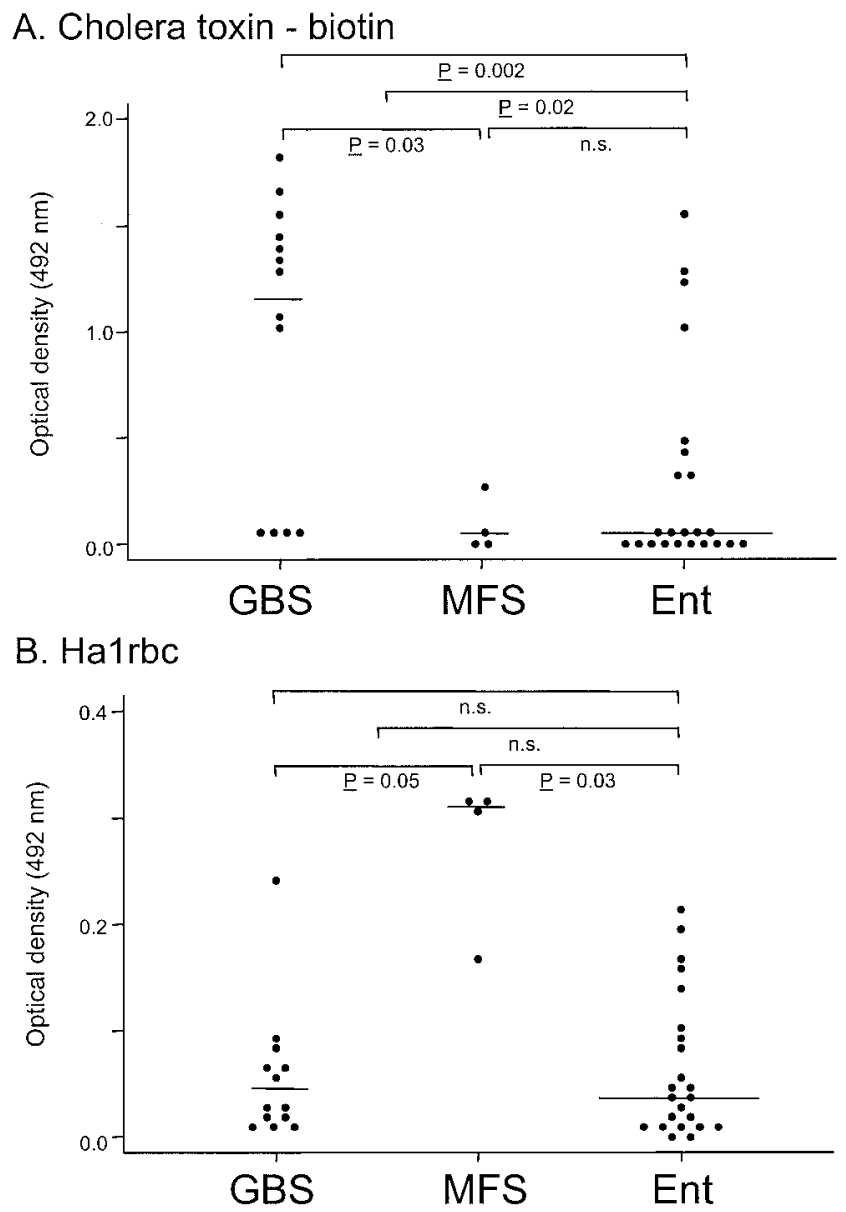

FIG. 3. Differential binding of CT and MAb Ha1rbc to C. jejuni LPS from patients with GBS, MFS, and uncomplicated enteritis (Ent). Purified C. jejuni LPS was coated onto 96-well plates and incubated with CT (biotin labeled) (A) or Ha1rbc (B). The differences in binding were compared between GBS and MFS isolates and between neuropathy-associated and uncomplicated enteritis isolates.

CT and the GM1 panel may recognize GM1-like immunoreactive epitopes on $C$. jejuni LPS and not necessarily an exact structural mimic of the GM1 oligosaccharide. Furthermore, our results indicate that approaches with the serum panel, toxins and lectins, and monoclonal antibodies all have their advantages and drawbacks. The observed good correlation between the three methods lead us to conclude that all three methods can be used to define "GM1-like" and "GQ1b-like" epitopes.

In conclusion, our data provide strong support for the hypothesis that molecular mimicry between $C$. jejuni LPS and gangliosides plays a key role in the induction of antiganglioside antibodies and neurological symptomes in patients with GBS or MFS. Furthermore, we have demonstrated that heterogeneity in the LPS structure determines the specificity of the antiglycolipid response and thereby the clinical features in patients with a post-Campylobacter neuropathy. The development of serological assays for the determination of ganglioside mimicry in C. jejuni LPS may be of great help in determining genetic markers for potentially neuropathic strains (27).

\section{ACKNOWLEDGMENTS}

This study was supported by grants from the Prinses Beatrix Fonds (95-0518) and The Netherlands Organization for Scientific Research (940-37-012) to C.W.A.

We thank P. Herbrink of the Diagnostic Centre SSDZ, Delft, The Netherlands, for performing Campylobacter infection serology and A. van Belkum for critically reading the manuscript.

\section{REFERENCES}

1. Ang, C. W., P. A. Van Doorn, H. P. Endtz, I. S. J. Merkies, B. C. Jacobs, M. A. De Klerk, R. Van Koningsveld, and F. G. A. Van der Meché. 2000. A case of Guillain-Barré syndrome following a family outbreak of Campylobacter jejuni enteritis. J. Neuroimmunol. 111:229-233.

2. Ang, C. W., N. Yuki, B. C. Jacobs, M. Koga, P. A. Van Doorn, P. I. M. Schmitz, and F. G. A. Van Der Meché. 1999. Rapidly progressive, predominantly motor Guillain-Barré syndrome with anti-GalNAc-GD1a antibodies. Neurology 53:2122-2127.

3. Angstrom, J., S. Teneberg, and K. A. Karlsson. 1994. Delineation and comparison of ganglioside-binding epitopes for the toxins of Vibrio cholerae, Escherichia coli, and Clostridium tetani: evidence for overlapping epitopes. Proc. Natl. Acad. Sci. USA 91:11859-11863.

4. Asbury, A. K., and D. R. Cornblath. 1990. Assessment of current diagnostic criteria for Guillain-Barré syndrome. Ann. Neurol. 27:S21-S24.

5. Aspinall, G. O., S. Fujimoto, A. G. McDonald, H. Pang, L. A. Kurjanczyk, and J. L. Penner. 1994. Lipopolysaccharides from Campylobacter jejuni associated with Guillain-Barré syndrome patients mimic human gangliosides in structure. Infect. Immun. 62:2122-2125.

6. Aspinall, G. O., C. M. Lynch, H. Pang, R. T. Shaver, and A. P. Moran. 1995. Chemical structures of the core region of Campylobacter jejuni O:3 lipopolysaccharide and an associated polysaccharide. Eur. J. Biochem. 231:570-578.

7. Endtz, H. P., C. W. Ang, N. Van Den Braak, B. Duim, A. Rigter, L. J. Price, D. L. Woodward, F. G. Rodgers, W. M. Johnson, J. A. Wagenaar, B. C. Jacobs, H. A. Verbrugh, and A. Van Belkum. 2000. Molecular characterization of Campylobacter jejuni from patients with Guillain-Barré and Miller Fisher syndromes. J. Clin. Microbiol. 38:2297-2301.

8. Goodyear, C. S., G. M. O'Hanlon, J. J. Plomp, E. R. Wagner, I. Morrison, J. Veitch, L. Cochrane, R. W. Bullens, P. C. Molenaar, J. Conner, and H. J. Willison. 1999. Monoclonal antibodies raised against Guillain-Barré syndrome-associated Campylobacter jejuni lipopolysaccharides react with neuronal gangliosides and paralyze muscle-nerve preparations. J. Clin. Investig. 104:697-708.

9. Herbrink, P., H. A. Van den Munckhof, M. Bumkens, J. Lindeman, and W. C. Van Dijk. 1988. Human serum antibody response in Campylobacter jejuni enteritis as measured by enzyme-linked immunosorbent assay. Eur. J. Clin. Microbiol. Infect. Dis. 7:388-393.

10. Jacobs, B. C., H. P. Endtz, F. G. A. Van der Meché, M. P. Hazenberg, H. A. M. Achtereekte, and P. A. Van Doorn. 1995. Serum anti-GQ1b IgG antibodies recognize surface epitopes on Campylobacter jejuni from patients with Miller Fisher syndrome. Ann. Neurol. 37:260-264.

11. Jacobs, B. C., H. P. Endtz, F. G. A. Van der Meché, M. P. Hazenberg, M. A. De Klerk, and P. A. Van Doorn. 1997. Humoral immune response against Campylobacter jejuni lipopolysaccharides in Guillain-Barré and Miller Fisher syndrome. J. Neuroimmunol. 79:62-68.

12. Jacobs, B. C., M. P. Hazenberg, P. A. Van Doorn, H. P. Endtz, and F. G. A. Van der Meché. 1997. Cross-reactive antibodies against gangliosides and Campylobacter jejuni lipopolysaccharides in patients with Guillain-Barré or Miller Fisher syndrome. J. Infect. Dis. 175:729-733.

13. Jacobs, B. C., P. H. Rothbarth, F. G. A. Van der Meché, P. Herbrink, P. I. M. Schmitz, M. A. De Klerk, and P. A. Van Doorn. 1998. The spectrum of antecedent infections in Guillain-Barré syndrome: a case-control study. Neurology 51:1110-1115.

14. Jacobs, B. C., P. A. Van Doorn, P. I. M. Schmitz, A. P. Tio-Gillen, P. Herbrink, L. H. Visser, H. Hooijkaas, and F. G. A. Van der Meché. 1996. Campylobacter jejuni infections and anti-GM1 antibodies in Guillain-Barré syndrome. Ann. Neurol. 40:181-187.

15. Kusunoki, S., A. Chiba, and I. Kanazawa. 1999. Anti-GQ1b IgG antibody is associated with ataxia as well as ophthalmoplegia. Muscle Nerve 22:10711074 .

16. Linton, D., M. Gilbert, P. G. Hitchen, A. Dell, H. R. Morris, W. W. Wakarchuk, N. A. Gregson, and B. W. Wren. 2000. Phase variation of a beta-1,3 galactosyltransferase involved in generation of the ganglioside GM1-like lipo-oligosaccharide of Campylobacter jejuni. Mol. Microbiol. 37:501-514.

17. Moran, A. P. 1997. Structure and conserved characteristics of Campylobacter jejuni lipopolysaccharides. J. Infect. Dis. 176:S115-121.

18. Nachamkin, I., H. Ung, A. P. Moran, D. Yoo, M. M. Prendergast, M. A Nicholson, K. Sheikh, T. Ho, A. K. Asbury, G. M. McKhann, and J. W. Griffin. 1999. Ganglioside GM1 mimicry in Campylobacter strains from sporadic infections in the United States. J. Infect. Dis. 179:1183-1189.

19. Nam Shin, J. E., S. Ackloo, A. S. Mainkar, M. A. Monteiro, H. Pang, J. L. Penner, and G. O. Aspinall. 1998. Lipo-oligosaccharides of Campylobacter 
jejuni serotype O:10: structures of core oligosaccharide regions from a bacterial isolate from a patient with the Miller-Fisher syndrome patient and from the serotype reference strain. Carbohydr. Res. 305:223-232.

20. Neisser, A., H. Bernheimer, T. Berger, A. P. Moran, and B. Schwerer. 1997. Serum antibodies against gangliosides and Campylobacter jejuni lipopolysaccharides in Miller Fisher syndrome. Infect. Immun. 65:4038-4042.

21. Paterson, G., G. Wilson, P. G. Kennedy, and H. J. Willison. 1995. Analysis of anti-GM1 ganglioside IgM antibodies cloned from motor neuropathy patients demonstrates diverse $\mathrm{V}$ region gene usage with extensive somatic mutation. J. Immunol. 155:3049-3059.

22. Prendergast, M. M., A. J. Lastovica, and A. P. Moran. 1998. Lipopolysaccharides from Campylobacter jejuni O:41 strains associated with GuillainBarré syndrome exhibit mimicry of GM1 ganglioside. Infect. Immun. 66: 3649-3655.

23. Rees, J. H., S. E. Soudain, N. A. Gregson, and R. A. Hughes. 1995. Campylobacter jejuni infection and Guillain-Barré syndrome. N. Engl. J. Med. 333:1374-1379.

24. Sack, D. A., A. J. Lastovica, S. H. Chang, and G. Pazzaglia. 1998. Microtiter assay for detecting Campylobacter spp. and Helicobacter pylori with surface gangliosides which bind cholera toxin. J. Clin. Microbiol. 36:2043-2045.

25. Salloway, S., L. A. Mermel, M. Seamans, G. O. Aspinall, J. E. Nam Shin, L. A. Kurjanczyk, and J. L. Penner. 1996. Miller-Fisher syndrome associated with Campylobacter jejuni bearing lipopolysaccharide molecules that mimic human ganglioside GD3. Infect. Immun. 64:2945-2949.

26. Sheikh, K. A., I. Nachamkin, T. W. Ho, H. J. Willison, J. Veitch, H. Ung, M. Nicholson, C. Y. Li, H. S. Wu, B. Q. Shen, D. R. Cornblath, A. K. Asbury, G. M. McKhann, and J. W. Griffin. 1998. Campylobacter jejuni lipopolysaccharides in Guillain-Barré syndrome: molecular mimicry and host susceptibility. Neurology 51:371-378.

27. Van Belkum, A., N. Van den Braak, P. Godschalk, W. Ang, B. Jacobs, M. Gilbert, W. Wakarchuk, H. Verbrugh, and H. Endtz. 2001. A Campylobacter jejuni gene associated with immune-mediated neuropathy. Nat. Med. 7:752753.

28. Wassenaar, T. M., M. Engelskirchen, S. Park, and A. Lastovica. 1997.
Differential uptake and killing potential of Campylobacter jejuni by human peripheral monocytes/macrophages. Med. Microbiol. Immunol. 186:139-144.

29. Willison, H. J., and G. M. O'Hanlon. 1999. The immunopathogenesis of Miller Fisher syndrome. J. Neuroimmunol. 100:3-12.

30. Willison, H. J., G. M. O'Hanlon, G. Paterson, J. Veitch, G. Wilson, M. Roberts, T. Tang, and A. Vincent. 1996. A somatically mutated human antiganglioside IgM antibody that induces experimental neuropathy in mice is encoded by the variable region heavy chain gene, V1-18. J. Clin. Investig. 97:1155-1164.

31. Willison, H. J., G. Paterson, P. G. Kennedy, and J. Veitch. 1994. Cloning of human anti-GM1 antibodies from motor neuropathy patients. Ann. Neurol. 35:471-478.

32. Yuki, N. 1997. Molecular mimicry between gangliosides and lipopolysaccharides of Campylobacter jejuni isolated from patients with Guillain-Barré syndrome and Miller Fisher syndrome. J. Infect. Dis. 176:S150-153.

33. Yuki, N., C. W. Ang, M. Koga, B. C. Jacobs, P. A. Van Doorn, K. Hirata, and F. G. A. Van der Meché. 2000. Clinical features and response to treatment in Guillain-Barré syndrome associated with antibodies to GM1b ganglioside. Ann. Neurol. 47:314-321.

34. Yuki, N., S. Handa, T. Tai, M. Takahashi, K. Saito, Y. Tsujino, and T. Taki. 1995. Ganglioside-like epitopes of lipopolysaccharides from Campylobacter jejuni (PEN 19) in three isolates from patients with Guillain-Barré syndrome. J. Neurol. Sci. 130:112-116.

35. Yuki, N., T. Taki, and S. Handa. 1996. Antibody to GalNAc-GD1a and GalNAc-GM1b in Guillain-Barré syndrome subsequent to Campylobacter jejuni enteritis. J. Neuroimmunol. 71:155-161.

36. Yuki, N., T. Taki, F. Inagaki, T. Kasama, M. Takahashi, K. Saito, S. Handa, and T. Miyatake. 1993. A bacterium lipopolysaccharide that elicits GuillainBarré syndrome has a GM1 ganglioside-like structure. J. Exp. Med. 178: 1771-1775.

37. Yuki, N., T. Taki, M. Takahashi, K. Saito, H. Yoshino, T. Tai, S. Handa, and T. Miyatake. 1994. Molecular mimicry between GQ1b ganglioside and lipopolysaccharides of Campylobacter jejuni isolated from patients with Fisher's syndrome. Ann. Neurol. 36:791-793.

Editor: R. N. Moore 